\title{
THE EFFECT OF CORE STABILITY TRAINING ON DYNAMIC BALANCE AND LOWER EXTREMITY PERFORMANCE IN YOUNG, ASYMPTOMATIC INDIVIDUALS
}

\author{
Maitri Modi *1, Geeta Bhatt ${ }^{2}$.
}

${ }^{{ }^{*} 1}$ Research Intern, K.J. Somaiya College Of Physiotherapy, Everard Nagar, Sion, Mumbai, India.

${ }^{2}$ Associate Professor, K.J. Somaiya College Of Physiotherapy, Everard Nagar, Sion, Mumbai, India.

\section{ABSTRACT}

Background and Purpose: Lower extremity performance and dynamic balance are an essential component of normal daily activities such as walking, running and climbing stairs. Thus, they are a key component of injury prevention and rehabilitation in sports. Training the core muscles has been hypothesized as an intervention for improving balance and lower extremity performance. However, there is a lack of current scientific evidence to support this claim. Hence, it is essential to verify the effects of the core stability training on dynamic balance and performance of lower extremity in young, asymptomatic individuals.

Materials and Methods: 40 young, asymptomatic individuals ( $n=20$ control, $n=20$ experimental) were selected and pre training sessions were held for both the groups in which limb length, core stability, dynamic balance using Star Excursion Balance Test and lower extremity performance using $T$ Test of agility were assessed. The subjects in the experimental group had to undergo progressive core stability training programme for 6 weeks and control group had to refrain from any form of structured core stability training for 6 weeks. After six weeks, both groups had to undergo post training session where all the parameters were assessed again.

Result: Data analysis revealed a significant increase $(p<0.0001)$ in the core stability and dynamic balance in the experimental group as compared to control group. There was a significant increase $(p<0.0001)$ in the lower extremity performance in both the groups individually. Whereas, there was no significant improvement $(p=0.05)$ in the lower extremity performance on comparing the control and experimental groups.

Conclusion: The study suggested that core stability training improves dynamic balance but failed to find a significant effect of core stability training on lower extremity performance.

Clinical Implications: Core stability training can be used in patients with balance deficits so as to improve balance and reduce the risk of falls and injuries.

KEY WORDS: Core stability, Dynamic balance, Lower extremity performance, $t$ test of agility, Modified star excursion balance test.

Address for correspondence: Ms. Maitri Modi, Research intern, K.J.Somaiya College of Physiotherapy, Everard Nagar, Sion, Mumbai-400022, India. E-Mail: maitrimod@gmail.com

\section{Access this Article online}

Quick Response code

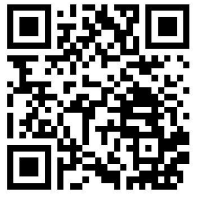

DOI: $10.16965 /$ ijpr.2017.227

International Journal of Physiotherapy and Research

ISSN 2321- 1822

www.ijmhr.org/ijpr.html

Received: 30-08-2017

Peer Review: 02-09-2017

Revised: 19-09-2017
Accepted: 19-10-2017

Published (0): 11-11-2017

Published (P): 11-12-2017

\section{INTRODUCTION}

Dynamic balance is the ability to maintain stability while in motion or in movement of the body or part of the body from one point to another and in maintaining stability. It causes the centre of gravity to move in response to muscular activity. This muscular activity may happen through any source of external or internal disturbance. During dynamic activity, the centre-of-pressure moves between the base of support boundaries and sometimes outside the base of support [1]. 
Lower extremity performance means measure of leg power, speed and agility. According to Kibler, core stability is defined as "the ability to control the position and motion of the trunk over the pelvis to allow optimum production, transfer, and control of force and motion to the terminal segment in integrated athletic activities [2]. The function of core musculature is better explained by dividing the trunk muscles into local and global categories. Local muscles are defined as those attaching to the lumbar vertebrae and influencing inter-segmental motion, while global muscles attach to the hips and pelvis and provide mobility and proper orientation of the spine [3]. The abdominal muscles, consisting of the transversus abdominis, rectus abdominis, and internal and external obliques, are involved primarily in controlling the position of the spine and pelvis [3]. It is believed that the core is important because of its anatomical location in the body where centre of gravity is located, thus where the movement stems. It functions to maintain the postural alignment and dynamic postural equilibrium during functional activities, which help avoid serial distortion patterns. The core musculature involves the muscles of the trunk and the pelvis that are responsible for maintaining the stability of the spine and pelvis. Thus, it acts as a base for motion of the distal segments, or "proximal stability for distal mobility" [2]. Hence, it can be said that the stability of the core musculature is responsible for optimizing the functioning/performance of lower extremity and improved balance.

\section{MATERIALS AND METHODS}

The study design was Experimental (Randomized control trial) conducted in the research lab of K.J.Somaiya College of Physiotherapy. 40 young, asymptomatic individuals $(\mathrm{N}=40)$ within the age group 18-30 years were selected provided that they did not have history of low back pain in last 6 months, any musculoskeletal/ neurological disorder and did not participate in any training program for strength training, core stability training, balance training. Verbal consent was taken from all. Simple Random Sampling method was done and the subjects were divided into two groups -Control $(n=20)$ and Experimental $(n=20)$. Pre training sessions were held for both the groups in which limb length, core stability, dynamic balance and lower extremity performance were assessed.

Dynamic Balance was assessed using modified Star Excursion Balance Test (mSEBT):

\section{- Anterior direction}

Fig. 1: Showing the participant performing the anterior component of mSEBT.

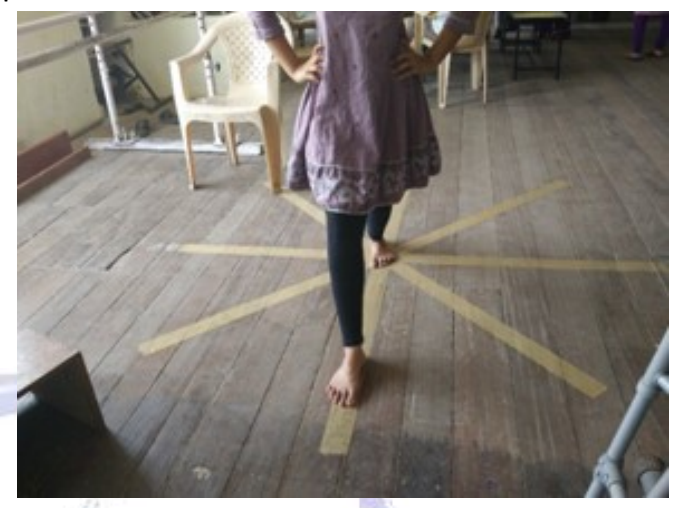

- Posteromedial Direction

Fig. 2: Showing the participant performing the posteromedial component of SEBT.

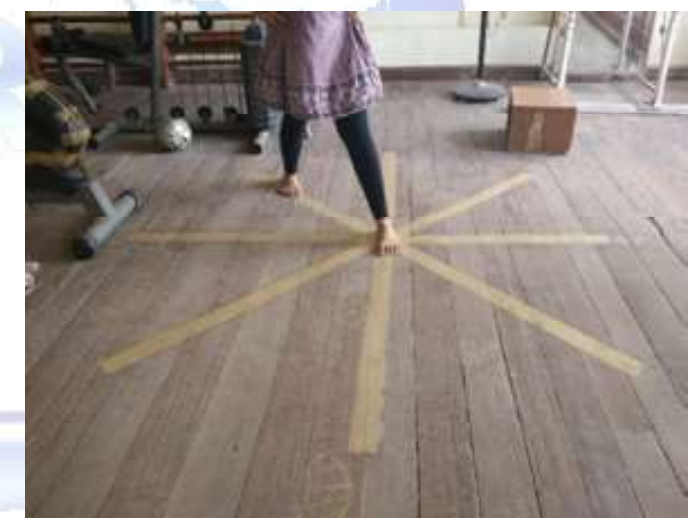

- Posterolateral Direction

Fig. 3: Showing the participant performing the posterolateral component of SEBT.

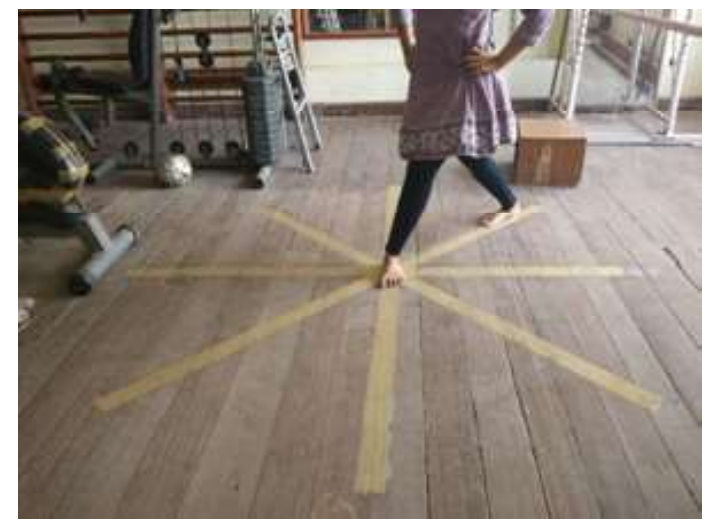

Participants performed the MSEBT standing at the centre of the grid laid on the floor with three reach lines in the form of $Y$. The reach lines were labelled in relation to the stance leg as anterior (A), posteromedial (PM) and posterolateral $(\mathrm{PL})$ directions extending at 135 degrees 
from each other [4]. The participants were asked to establish a stable base of support on the stance limb in the middle of the testing grid and maintain it through a maximal reach excursion in one of the prescribed directions. They then had to reach as far as possible along each of the three lines, make a light touch on the line with the most distal part of the big toe and return the reaching leg back to the center while maintain a single-leg stance with the other leg. Each participant performed three trials in each direction on each leg. Ten seconds of rest were provided between the different trials of one reach direction.

A trial was discarded and repeated if participants

1. took weight on the reaching foot

2. failed to bring back the reaching foot to starting position

3. failed to keep both hands on the hips

4. failed to keep the stance foot in place or

5. failed to keep the forefoot or heel of the stance foot on the floor. [4]

Lower Extremity Performance was assessed using $T$ Test of agility [5].

Fig. 4:

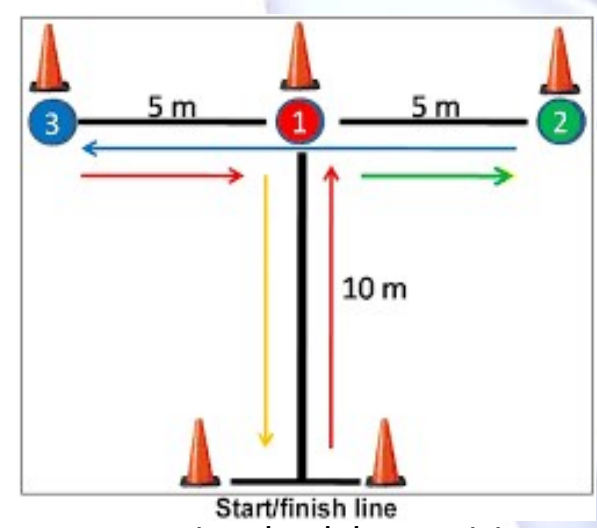

This assessment involved the participant to move in a T-shaped pattern. At the signal of ' $G O$ ', the participant commenced the test. He/she then ran straight ahead from the cone at the base of the ' $T$ ' and touched the cone in the centre, side stepped 5 metres to the left cone and touched it, side stepped 10 metres to the far cone to the right and touched it, side stepped 5 metres to the middle cone and touched it and then ran 10 metres backwards to the base of the ' $T$ ' and touched that cone. The time taken to complete the test was recorded. 2 practice runs were given prior to the final test with rest period of $2 \mathrm{~min}$ utes in between.

After squaring the pelvis, limb length was measured using a measuring tape. This was done to normalize the reach distance in $\mathrm{MSEBT}$ (excursion distance/leg length*100).

Core stability was assessed using pressure biofeed back unit using the PRONE method and calculating the number of times for which the subject could hold the contraction for 10 seconds [6].The subjects in the experimental group then had to undergo progressive core stability training programme for 6 weeks and control group had to refrain from any form of structured core stability training for 6 weeks but continue with all their other daily activities. After six weeks, both groups had to undergo post training session where core stability, dynamic balance and lower extremity performance were assessed again.

Core stability exercises: Crook lying with abdominal hollowing, Kneeling with abdominal hollowing, Quadruped with one leg raise and abdominal hollowing, Quadruped with opposite leg and arm raise with abdominal hollowing, Kneel sitting to kneeling with abdominal hollowing, Squatting with abdominal hollowing, Ball sitting with one leg raise and abdominal hollowing, Roll ball in and out with abdominal hollowing, Air cycling with abdominal hollowing, Side plank, Prone plank.

Table 1: Core stability exercise progression.

\begin{tabular}{|c|c|c|c|c|c|c|}
\hline $\begin{array}{c}\text { Week (sec*time) } \\
\text { EXERCISES }\end{array}$ & 1 & 2 & 3 & 4 & 5 & 6 \\
\hline 1 & 5 secs $* 5$ & 10 secs $* 5$ & 15 secs $* 10$ & 20 secs $* 10$ & 25 secs $* 15$ & 30 secs*15 \\
\hline 2 & $5 \operatorname{secs} * 5$ & 10 secs $* 5$ & 15 secs $* 10$ & 20 secs $* 10$ & 25 secs $* 15$ & 30 secs $* 15$ \\
\hline 3 & $5 \operatorname{secs} * 5$ & 10 secs $* 5$ & 15 secs $* 10$ & 20 secs $* 10$ & 25 secs $* 15$ & 30 secs $* 10$ \\
\hline 4 & $5 \operatorname{secs} * 5$ & 10 secs*5 & 15 secs $* 10$ & 20 secs $* 10$ & 25 secs $* 15$ & 30 secs $* 10$ \\
\hline 5 & $5 \operatorname{secs} * 3$ & 10secs*3 & 10 secs $* 5$ & 15 secs $* 10$ & $20 \operatorname{secs} * 10$ & 25 secs $* 15$ \\
\hline 6 & $5 \operatorname{secs} * 3$ & 10 secs*3 & 10 secs $* 5$ & 15 secs $* 10$ & 20 secs $* 10$ & 25 secs $* 15$ \\
\hline 7 & $3 \operatorname{secs} * 3$ & 10 secs $* 3$ & 10 secs*5 & 15 secs $* 10$ & $20 \operatorname{secs} * 10$ & 25 secs $* 15$ \\
\hline 8 & $3 \operatorname{secs} * 3$ & 5 secs*5 & 10 secs $* 5$ & 10 secs $* 10$ & 15 secs $* 10$ & 15 secs*15 \\
\hline 9 & $3 \operatorname{secs} * 3$ & 5 secs $* 5$ & 10 secs*5 & 10 secs $* 10$ & 15 secs $* 10$ & 15 secs $* 15$ \\
\hline 10 & $3 \operatorname{secs} * 3$ & 5 secs $* 5$ & 10 secs $* 5$ & 10 secs $* 10$ & 15 secs $* 10$ & 15 secs $* 15$ \\
\hline 11 & $3 \operatorname{secs}^{*} 3$ & 5 secs $* 5$ & 10 secs*5 & 10 secs $* 10$ & $15 \operatorname{secs} * 10$ & 15 secs $* 15$ \\
\hline
\end{tabular}

\section{RESULTS}

The data was entered and analyzed using Graphpad instat 3. The data was numerical, for 
data which passed the normality test, parametric tests were used and for data which did not pass the normality test, non parametric tests were used. Paired t test/Wilcoxon matched test was used for comparison within the two groups (experimental and control). Unpaired t test/ Mann Whitney test was used for comparison between the two groups. Significance level was set at $95 \%$ confidence level for all statistical tests.

On comparison of core stability between Experimental and Control groups, the $p$ value was $<0.0001$, which was considered extremely significant.

On comparison of dynamic balance between Experimental and Control groups, the $p$ value was considered significant for all the 3 components.

On comparison of t test of agility between Experimental and Control groups, the $p$ value was 0.054 , which was considered not quite significant.

Graph 1: Comparison of core stability in both the groups.

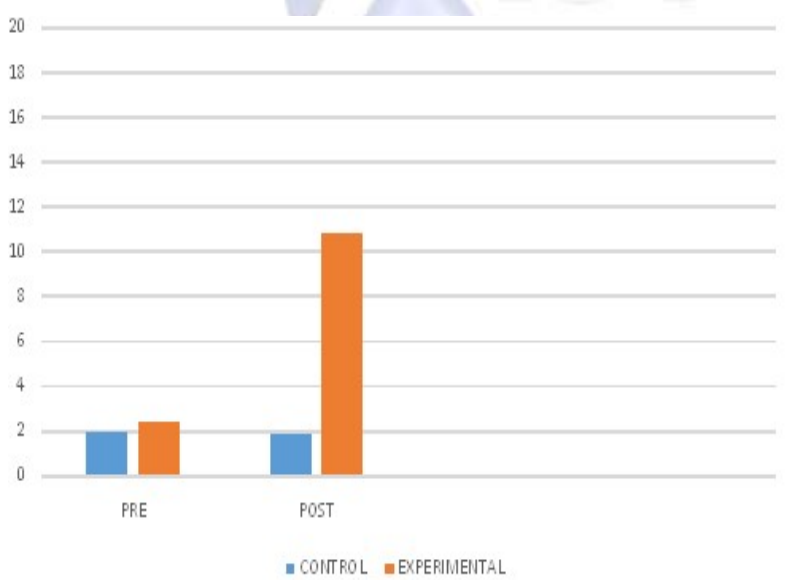

Graph 2: Comparison of excursion distances (Left and Right Anterior directions) in both groups.

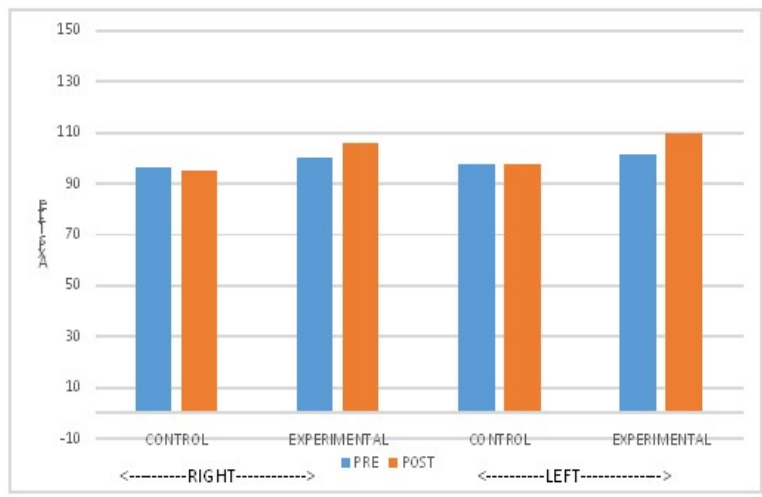

Graph 3: Comparison of excursion distances (Left and Right Posteromedial directions) in both groups.

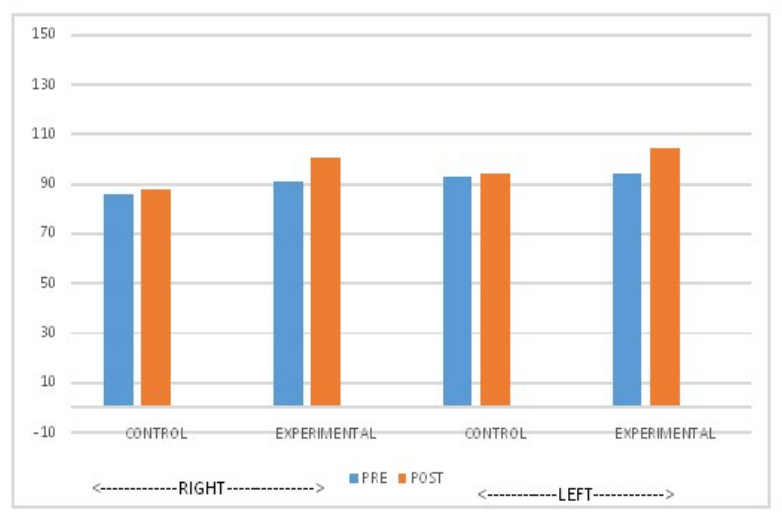

Graph 4: Comparison of excursion distances (Left and Right Posterolateral directions) in both groups.

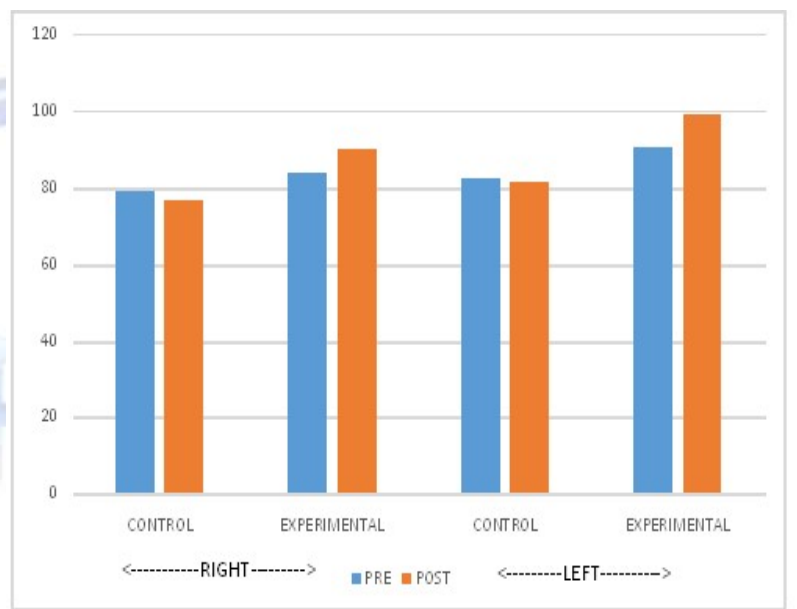

Graph 5: Comparison of lower extremity performance ( $T$ test) in both the groups.

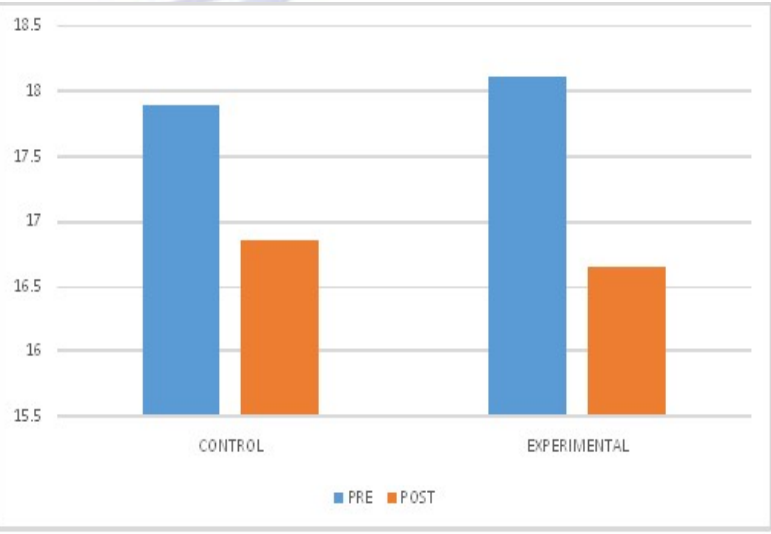

\section{DISCUSSION}

The purpose of the study was to evaluate the effects of six weeks of core stability training programme on dynamic balance and lower extremity performance in healthy, young adults. The results of the present study revealed that: There was significant improvement in core stability in experimental group as compared to control group following core stability training programme. 
There was significant improvement in all three components of $\mathrm{mSEBT}$ for dynamic balance in experimental group as compared to control group following core stability training programme.

There was significant improvement in T test of agility for lower extremity performance in experimental and control groups following core stability training programme.

There was no significant improvement in the lower extremity performance on comparing the control and experimental groups.

For the three reach directions, normalized maximum excursion distances increased significantly from pre to post test for the experimental group. This improvement in reach distance verifies that core stability does have an effect on dynamic balance. As stated by Kibler et al.[2], the larger muscles of the core create a rigid cylinder and a greater moment of inertia against body perturbation while allowing a stable base for mobility. The abdominal muscles comprising of the transverse abdominis, internal and external obliques and rectus abdominis all contract to provide stabilization for the spine and hence, a stronger base of support for lower extremity movement [2]. The transversus abdominis has also been shown to be critical in stabilization of the lumbar spine. When it contracts, the intra-abdominal pressure increases and the thoracolumbar fascia tenses [2]. These contractions occur before initiation of limb movement which allow the limbs to have a stable base for motion and muscle activation [7]. The rectus abdominis and oblique abdominals are activated in direction specific patterns with respect to the limb movements, thus they provide postural support before limb movements [2]. Richardson proposed that a precise co-contraction of the transversus abdominis and multifidus are independent of the global musculature, neutral spine posture. This feed forward nature of activation increases muscle stiffness and segmental stabilization to provide more efficient use of the primary muscles. Consequently, delayed onset of transversus abdominis activation leads to inefficient muscular stabilization of the spine and compromised balance [7].

There is no specific protocol for a core training programme. Overall, the exercises need to concentrate on motor control, emphasizing the neutral spine posture, contraction of the pelvic floor muscles and transversus abdominis with the multifidus. The exercises included in this study progressed from a large base of support to small base of support and from stable surface to unstable surface.

According to Kibler et al., the body uses core muscle activation to generate the necessary rotational torques around the body and produce extremity motion [2]. So, in mSEBT, as the subject stands on the stance leg and uses the opposite limb to reach, the rectus abdominis muscles and obliques would fire before the movement occurs to perform trunk motion, which allows the subject to maintain balance. Also, the multifidi and transverse abdominis muscles would help to maintain dynamic balance during lower extremity movement by providing support to the lumbar spine [8].

For this study, only three of the possible eight star excursion directions were used for statistical analysis consisting of the Anteromedial, Medial and Posteromedial directions. These three were chosen based on recent work that has demonstrated that the eight directions of the SEBT may be paired down for efficiency of task evaluation because of redundancy across the reaching directions. The number of reach directions administered could be decreased without compromising the validity of the test. In this study, the three reaching directions all were sensitive at demonstrating that dynamic balance improved by core stability training [9]. It is essential to note that while male and females were included in the study, there was no significant influence of sex on the measures. As reported by Gribble and Hertel, gender differences may be controlled by normalizing the raw reaching distance to the leg length of the stance limb; a procedure that we followed in our study [10]. Our study failed to find any significant effect of core stability training on lower extremity performance. Although there was a significant improvement in the $T$ test of agility pre and post in both the groups individually, but there was no in between group improvement. This can be because of the practice period which the subjects in both the groups obtained which led to increase in their 
confidence levels while performing the T test of agility, thus leading to significant improvements in pre and post test timings in both groups.

Also, according to Stanton, specificity of exercise selection should be considered [11]. The exercise regimen in our study focuses more on dynamic balance and core stability and less on speed, agility and power.

According to Reed CA [12], in an athletic development programme core stability is rarely the sole component, thus it is difficult to directly isolate its effect on athletic performance. Conflicting findings and the lack of a standardization for measurement of outcomes and training focused to improve core strength and stability pose difficulties. Because of this, further research targeted to determine this relationship is necessary to better understand how core strength and stability affect lower extremity performance [12].

Clinical Implication: Core stability training can be used in patients with balance deficits so as to improve balance and reduce risk of fall and injuries, thus leading to a better quality of life.

A clearer understanding of the roles that specific muscles have during core stability and core strength exercises would enable more functional training programmes to be implemented, which may result in a more effective transfer of these skills to actual sporting activities.

\section{CONCLUSION}

Thus, the study concluded that core stability training has a significant effect on dynamic balance. But, the study failed to find significant effect of core stability training on lower extremity performance in young, asymptomatic individuals.

\section{Conflicts of interest: None}

\section{REFERENCES}

[1]. Hassan Sadeghi, Ardalan Shariat, Enayatollah Asadmanesh, Maryam Mosavat. The Effects of Core Stability Exercise on the Dynamic Balance of Volleyball Players. International Journal of Applied Exercise Physiology 2013;2(2);Page 2.
[2]. W. Ben Kibler, Joel Press and Aaron Sciasci.The Role of Core Stability in Athletic Function.Sports Med 2006; 36 (3): 189-198.

[3]. Chris Sharrock, Jarrod Cropper, Joel Mostad,Matt Johnson, and Terry Malone. A pilot study of core stability and athletic performance: is there a relationship? International Journal of Sports Physical Therapy. 2011 Jun; 6(2): 63-74.

[4]. Remko van Lieshout. Reproducibility of the modified star excursion balance test composite and specific reach direction scores, IJSPT; June 2016; 11(3).

[5]. Kainoa Pauloe, Kent Madole, John Garhammer, Michael Lacourse and Ralph Rozenek. Reliability and Validity of the T-Test as a Measure of Agility, Leg Power, and Leg Speed in College-Aged Men and Women. Journal of Strength and Conditioning Research, 2000, 14(4), 443-450.

[6]. Carolyn Kisner, Lynn Allen Colby. Therapeutic Exercise, Foundation and Techniques. Sixth edition; Page 512.

[7]. Sheri P. Silfies, Rupal Mehta, Sue S. Smith, Andrew R. Karduna. Differences in Feedforward Trunk Muscle Activity Subgroups of Patients With Mechanical Low Back Pain. Arch Phys Med Rehabil 2009; 90:115969.

[8]. Nicole L. Kahle, BS; Phillip A. Gribble. The effects of core stability training on balance testing in young, healthy adults. Athletic Training and Sports Health Care March/April 2009 - Volume 1 · Issue 2: 65-73.

[9]. Robinson R, Gribble P. Support for a reduction in the number of trials needed for the Star Excursion Balance Test. Arch Phys Med Rehabil. 2008; 89(2):364370.

[10]. Gribble P, Hertel J. Considerations for normalizing measures of the Star Excursion Balance Test. Measure Phys Educ Exerc Sci. 2003;7(2):89-100.

[11]. Stanton R, Reaburn PR, Humphries B. The effect of short-term Swiss ball training on core stability and running economy. J Strength Cond Res. 2004 Aug;18(3):522-8.

[12]. Reed CA1, Ford KR, Myer GD, Hewett TE. The effects of isolated and integrated 'core stability' training on athletic performance measures: a systematic review. Sports Med. 2012 Aug 1;42(8):697-706.

How to cite this article:

Maitri Modi, Geeta Bhatt. THE EFFECT OF CORE STABILITY TRAINING ON DYNAMIC BALANCE AND LOWER EXTREMITY PERFORMANCE IN YOUNG, ASYMPTOMATIC INDIVIDUALS. Int J Physiother Res 2017;5(6):2451-2456. DOI: 10.16965/ijpr.2017.227 